\title{
The description and analysis of conditioned suppression: A critique of the conventional suppression ratio
}

\author{
HARRY M. B. HURWITZ and HANK DAVIS \\ University of Guelph, Guelph, Ontario, Canada
}

\begin{abstract}
It is argued that the conventional $A /(A+B)$ ratio is an inappropriate tool for both the description and analysis of conditioned suppression. Conditioned suppression is a procedure that generates two separate dependent variables that cannot be meaningfully collapsed into a single index. Using a relatively simple experiment, we demonstrate that the procedure typically (1) suppresses responding during the CS and (2) generates a U-shaped function in the recovery of baseline responding. These reliable shifts in baseline rate confound the interpretation of the suppression ratio. It is further argued that comparison between individuals or groups of cases using an analysis of variance performed on suppression ratios is inappropriate because of numerous violations of the assumptions of the test. We offer a number of alternative strategies for the description and analysis of conditioned suppression. These include the use of raw data analyzed with nonparametric tests or an analysis of covariance, a procedure that accommodates the need for ipsative measurement without producing the flaws inherent in ANOVAs performed on suppression ratios.
\end{abstract}

Estes and Skinner (1941) performed the original experiment to study the effects of experimentally induced anxiety. They began by establishing a baseline of food-reinforced behavior in rats. In the next phase of the experiment, a 5-min tone (CS) was presented prior to the delivery of a brief inescapable electric shock (US). This procedure resulted in the reduction of food-reinforced responding in the presence of the preshock signal. The change in behavior was attributed to "anxiety," and like the procedure that generated it, it has since been referred to as conditioned suppression.

Virtually all of the early conditioned suppression research involves individual organism designs, as advocated by Skinner $(1938,1953)$ and Sidman (1960). Ironically, however, the original experiment by Estes and Skinner did not report data from individual subjects, but rather presented mechanically averaged cumulative response curves. Despite the fact that a fixed-interval 4-min schedule maintained responding, there were virtually no fluctuations from a steady rate of responding. These records, despite their syn-

This research was supported in part by Grant A7496 (Hurwitz) and A0673 (Davis) from the Natural Sciences and Engineering Research Council of Canada. The authors are indebted to Roland Chrisjohn for his expert statistical guidance and to Marc B. Hurwitz for his help in clarifying some mathematical issues. Our thanks are extended to John Horn for his comments on the penultimate draft of the manuscript. Glen Forster's technical assistance was essential to the successful completion of the experiment. Portions of this paper were presented at meetings of the Experimental Psychology Society, Cambridge, U.K., 1982, and the Psychonomic Society, Philadelphia, 1981. thetic nature, helped perpetuate the myth of the stable baseline, in which pre-CS responding remains essentially constant and is interrupted only by the occurrence of the conditioned aversive stimulus. Because it was believed that the effects of the procedure were confined to the presence of the CS, the most useful measure of the development of suppressive control would appear to be a ratio of responding during the CS relative to responding when the CS was absent. The suppression ratio was later elaborated by Kamin and his colleagues into its universally accepted $\mathbf{A} /(\mathbf{A}+\mathbf{B})$ form, in which $\mathbf{A}=$ responding during the $C S$ and $B=$ responding during the pre-CS, or baseline, portion of the session (e.g., Annau \& Kamin, 1961).

Despite its wide acceptance, the suppression ratio has an essential vulnerability. Because changes in $\mathbf{A}$ are measured relative to $B$, if the value of $B$ also begins to shift, interpretation of the ratio is obfuscated. Moreover, if one is interested in changes in the value of $B$, there is no conventionally accepted referent for its measurement. Arguably, the appropriate reference point for measuring changes in $B$ would be its origin, the prime, or pretreatment, rate of response.

It is widely assumed that the prime rate of responding $(P)$ is maintained despite introduction of CS-US pairings (Davis \& Hurwitz, 1977, p. 184). This may not be the case. In fact, there is considerable evidence that baseline responding itself is a major dependent variable of the conditioned suppression procedure (e.g., Baker, Mercier, Gabel, \& Baker, 1981; Davis, 1981; Davis \& MacIntire, 1969; Millenson \& Dent, 1971). 
Because such objections to the suppression ratio were not given explicit statement, and because of the convincing and robust nature of conditioned suppression itself, the procedure began to enjoy a wide range of application during the 1960 s. There are now over 1,000 studies in the literature which used the conditioned suppression procedure as the principal instrument of investigation (Blackman, 1977; Davis, 1968; Davis \& Wright, 1979; Hurwitz \& Roberts, 1977). Almost inevitably, conditioned suppression studies adopted conventional between-group designs in which the suppression ratio served as the unit of measurement. Between-group technology requires the use of inferential statistics to make comparisons and to establish the validity of independent variables. However, the most common statistical tests, such as the ubiquitous analysis of variance (ANOVA), are based on a set of assumptions which, as we shall argue, have been consistently violated by the adoption of a ratio as the dependent measure of conditioned suppression. In fact, the major point of this paper is that the conventional suppression ratio is a totally inappropriate tool for analyzing conditioned suppression. Not only does it violate the statistical assumptions necessary to make betweengroup comparisons, it is inadequate even in describing the behavioral effects of individual cases.

To illustrate some of our concerns with the conditioned suppression ratio, we have performed a relatively simple experiment. Animals were first trained to respond for food on a variable interval (VI) 1-min schedule of reinforcement. When responding had stabilized, one group of rats was given three CS-US trials per session; a second group received six CS-US trials per session. All subjects were run for 21 training sessions, followed by 10 extinction sessions, during which CS presentations were no longer followed by shock.

The decision to compare the effects of three versus six trials per session stems from suggestive evidence from our laboratory (Davis, Shattuck, \& Wright, 1981) that trial density does not affect CS control, although it may determine the degree of baseline suppression.

\section{METHOD}

\section{Subjects}

Twelve male Long-Evans rats, approximately 100 days old a: the start of training, served as subjects. They were individually housed with water freely available in their home cages, and were maintained at approximately $80 \%$ of their pretest weights.

\footnotetext{
Apparatus

Subjects were trained to leverpress and were tested in commerically available rodent test chambers (Campden Instrument Company Ltd.). Forty-five-milligram Noyes pellets served as reinforcers. Sixty-second tones (the CS) were provided by a Mallory Sonalert (Model SC-628-H) located above the test chamber and monitored in conjunction with background white noise at
}

$86 \mathrm{~dB}$ (re .0002 dynes $/ \mathrm{cm}^{2}$ ). Shock, the US, was delivered from a Grason-Stadler generator calibrated at $0.6 \mathrm{~mA}$ for $1 \mathrm{sec}$.

\section{Procedure}

Following preliminary leverpress training, all subjects were run for daily 1 -h sessions on a variable interval $60-\sec$ food reinforcement schedule. The cumulative response records revealed stable baseline responding after 24 sessions, which was confirmed by a coefficient of variation.

The subjects were divided into two groups $(N=6)$, which received either three or six tone-shock (CS-US) trials per session. Following 21 conditioned suppression sessions, the subjects were exposed to an extinction procedure for $101-\mathrm{h}$ sessions, during which the CS was presented but the US was withheld. Recordings were made of rates of responding before the CS-US procedure was introduced (prime rate); responding during the $2 \mathrm{~min}$ prior to and during each tone-shock interval on CS-US days; and responding 2 min prior to and during each CS on extinction days.

\section{RESULTS}

As we noted previously, there are three primary dependent measures of conditioned suppression: (1) the pretreatment, or prime rate, of variable-interval responding; (2) the baseline (pre-CS) rate of responding; and (3) the rate of responding during CS presentations. Before examining our research in terms of these three measures, it is worth noting that neither our procedure nor any of the stimulus parameters we employed were extraordinary. Rather, we used a conditioned suppression procedure that lies well within the range of general practice (Davis \& Wright, 1979).

\section{Pretreatment (Prime Rate) of VI responding}

Prior to exposure to the conditioned suppression procedure, there was considerable variability between the rates of individual subjects on the VI schedule. The mean rate of responding during the final five sessions ranged from 16 to 51 responses/ min. The mean rate for all subjects was 31.5. There was general stability within each subject's rate over the final sessions, as measured by a coefficient of variation $(\mathrm{CV}<.10)$. Prior to experimental treatment, the mean prime rate for Group 3 was 35.5 , and the mean prime rate for Group 6 was 27.5. These values did not differ.

\section{Baseline (Pre-CS) Rate of Response}

Figure 1 presents median baseline rates of response for both groups. These measures were obtained for $2-$ min periods prior to each CS in the session. Medians were chosen because of extreme between-subject variability. In fact, there were subjects whose baseline responding remained near zero for all 21 conditioned suppression sessions. This created an unusually large and nonhomogeneous variance, thus violating one of the basic requirements for the analysis of variance. For reasons to be elaborated later, we will restrict all subsequent between-group comparisons to nonparametric tests of significance. 


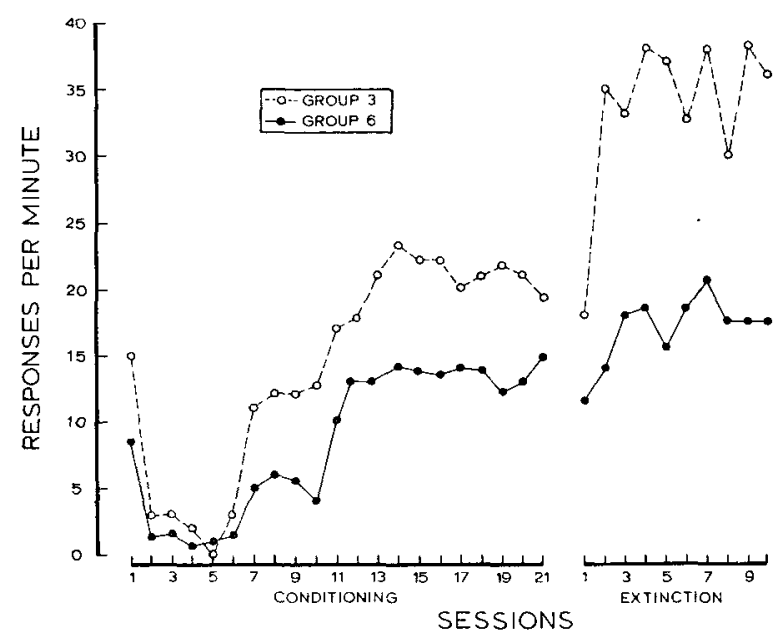

Figure 1. Median rate of responding during baseline (pre-CS) minutes obtained during 21 conditioned suppression sessions and during 10 extinction sessions in which shock was omitted. Group 3 received three CS-US trials per 1-h conditioning session; Group 6 recelved six trials per session.

Baseline response rates of all animals, regardless of group, were significantly reduced from prime rate ( $\mathrm{p}<.05$, randomization test). Figure 1 reveals a marked U-shaped function in the baseline rates for both groups over the first 12 sessions. This function is representative of all subjects except two in Group 3, whose baselines failed to recover. Nevertheless, the baseline response rates of subjects in Group 3 stabilized at higher asymptotic levels than those observed in Group 6 over the final 10 sessions ( $p<$ .001 , randomization test).

Although there was general recovery of baseline responding once the US was withheld, residual suppression remained throughout 10 extinction sessions for Group 6. There was a substantial increase in baseline responding above prime rate for Group 3 over

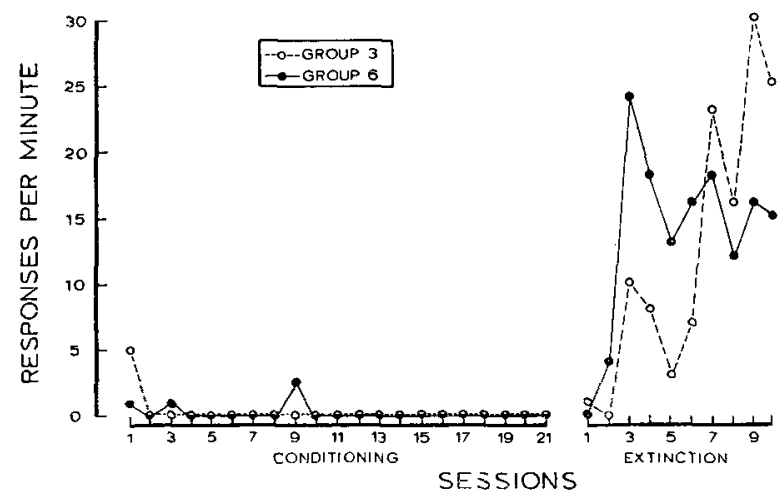

Flgure 2. Median rate of responding during CS minutes obtained during 21 conditioned suppression sessions, and 10 extinction sessions in which shock was omitted. Group 3 received three CS-US trials per 1-h conditioning session; Group 6 recelved six trials per session.
- the last 9 extinction sessions ( $p<.01$, sign test), despite the fact that the subjects in this group had received half the number of extinction trials.

The groups differed with respect to the degree of prime rate recovery. Responding by subjects in Group 3 was elevated over the prime rate, whereas a comparison between responding on the final 2 days of extinction and the prime rate revealed no difference for subjects in Group 6.

\section{Rate of Responding During CS}

Figure 2 presents the median response rates of both groups of animals during the CS. Responding in the presence of the CS fell to near zero in both groups following the first session and failed to recover over the next 20 sessions. When shock was withheld during extinction, responding resumed during the $\mathrm{CS}$ in all but one subject (Group 3). It is notable that despite their recovery of baseline responding beyond prime rate, subjects in Group 3 continued to show residual suppression during the CS. A within-subject comparison of CS and pre-CS response rates during the final two extinction sessions revealed a significant difference $[\mathrm{t}(11)=3.7, \mathrm{p}<.01]$, thereby indicating differential suppression to the CS. A similar analysis of responding by subjects in Group 6 revealed no residual suppression.

\section{DISCUSSION}

We have performed a relatively simple experiment and demonstrated that three versus six CS-US trials per session yields different effects within a conditioned suppression procedure. It is notable, however, that our results were neither reported nor analyzed in terms of the conventional $\mathbf{A} /(\mathbf{A}+\mathbf{B})$ suppression ratio. Rather, the effects of three versus six trials were primarily observed on the rate of baseline responding following recovery from the initial suppressive effects of the procedure. When we used the conventional suppression ratio, we found no difference between groups in either the rate of decline or the terminal ratio value. Although a group difference in suppression ratios did occur during extinction ( $p<.002$, sign test), this effect was no doubt attributable to the observed group difference in baseline rate during this same period.

Following collection of the present data, we reexamined other results from our laboratory. This survey suggests that certain features of the present outcome are typical: (1) Following one or two conditioned suppression sessions, response rate is reduced to near zero in both CS and pre-CS periods. For the majority of subjects there is recovery of response rate when the CS is absent, although this recovery is usually incomplete. In short, the prime rate is rarely recovered. The difference between baseline and $\mathbf{P}$ may itself be viewed as a primary dependent 
variable of conditioned suppression. (2) The change in baseline rate over sessions typically follows a $U$ shape, the duration and depth of which may relate to experimental parameters, such as shock duration, shock intensity, schedule of food reinforcement, etc. Such changes in baseline rate may be quite profound and their effects disruptive of both the development and analysis of conditioned suppression (Millenson \& Dent, 1971; Millenson \& DeVilliers, 1972).

\section{Implications of Baseline Changes}

Our finding that baseline response rates followed a U-shaped function over sessions has direct implications for the analysis of conditioned suppression. As we previously noted, interpretation of the suppression ratio is confounded until the value of $B$ recovers and stabilizes. However, our data suggest that this is unlikely to occur within the limited number of sessions commonly employed in studies of conditioned suppression (e.g., Ayers, Berger-Gross, Kohler, Mahoney, \& Stone, 1979; Dickinson, Hall, \& Mackintosh, 1976).

The initial decline in rate of responding has been observed by other experimenters. ${ }^{1}$ In fact, some have taken explicit steps to reduce its effects. Millenson and his colleagues found it necessary to reduce shock intensity or to give rehabilitation sessions in order to reinstate responding to a level that allowed meaningful interpretation of the suppression ratio. The technique of "baseline recovery sessions" has been adopted by other investigators as well (e.g., Baker \& Mackintosh, 1979; Baker \& Mercier, 1982), and it is not unusual to see subjects dropped from the experiment for failing to show baseline recovery (e.g., Dickinson, Colwill, \& Pearce, 1980).

In short, although severe baseline reduction appears to have been widely acknowledged, its potential effects on the suppression ratio do not seem to be fully appreciated. Our findings suggest not only an initial decline in response rate, but also the eventual recovery of responding without additional intervention. However, two points must be noted: (1) The level at which baseline rate restabilizes may still represent a significant change from prime rate, and (2) the U-shaped recovery function itself may differ between groups. Both of the points underscore the descriptive futility of the suppression ratio, not only when computed prior to restabilization of the baseline, but also after baselines have restabilized to unequal response rates.

\section{Descriptive Inadequacies of the Suppression Ratio}

It is widely accepted that conditioned suppression is essentially a relative phenomenon; that is, the degree of suppression during a CS is best appraised relative to what occurs immediately prior to the CS. At first glance it would seem that this problem might have been solved by using a difference score. For example, each subject's rate of responding during the CS might have been subtracted from its preCS rate. The likely reason that this technique was not adopted is that it would have generated considerable between-subject variability. In contrast, the use of a ratio appears to focus on relative differences while eliminating unwanted variability. It does this by providing a common origin for the measure; that is, 0.5 indicates equivalence between $\mathrm{CS}$ and pre-CS rates regardless of their absolute values.

However, the solution provided by the suppression ratio is illusory. There are at least two objections; we have previously suggested the first of these. Simply put, a ratio is uninterpretable when the values of both of its components are variable. It is unlikely that either component will remain fixed insofar as both the CS rate and the pre-CS rate are systematically changed by the conditioned suppression procedure. Inasmuch as the value of B (pre-CS baseline) is likely to vary, a reduction in the suppression ratio need not reflect reduced responding during the CS, as is commonly assumed.

With regard to the value of a component's being "known," a different case can be made against the suppression ratio. If, for example, knowledge of conditioned suppression were advanced to include the $U$ shaped function in baseline recovery, it would then be appropriate to use both dependent variables in the analysis of conditioned suppression. In short, at the very moment the suppression ratio becomes interpretable because its components are both known, it also becomes redundant.

There are numerous examples of the first of these infractions, not excluding work from our own laboratory (e.g., Davis \& Levine, 1982; Davis et al., 1981). In other examples, Randich and LoLordo (1979) examined the effects of shock preexposure by comparing the suppression ratios of groups of preexposed and nonpreexposed subjects. This comparison proceeded for only six test sessions; moreover, significant ratio differences were reported at the very time that significant differences were also reported in group baseline rates. Similarly, Dickinson et al. (1980) reported group differences in suppression ratios, while acknowledging significant group differences in baseline rates. Randich and Rescorla (1981) did the same, noting not only differences in pre-CS rates, but within-session shifts in baseline rate as well. Further evidence that baseline suppression is not seriously viewed as a dependent variable stems from cases of multiple experiments in which no note is taken of the fact that baseline rates differed between experiments (e.g., Baker \& Mackintosh, 1979; Rescorla, 1968).

Descriptive inadequacies in the suppression ratio are further illustrated in the following two cases: (1) If suppression of responding is total (i.e., not dif- 
ferentially conditioned to the CS), a ratio value of 0 is typically assigned (Annau \& Kamin, 1961). This ratio value obscures the fact that differentiation, a keynote of conditioned suppression, has not occurred. (2) If responding is severely, but not totally, disrupted throughout the entire session, including presentations of the CS, then a ratio value of 0.5 will result. This value would fail to indicate the magnitude of conditioned "fear," the central state which was our original concern (Estes \& Skinner, 1941).

There is a further descriptive inadequacy of the suppression ratio. It is commonly assumed that suppression ratio values, like the raw data they reflect, constitute ratio scale measurement. Moreover, it is implicitly assumed that the relation between suppression ratios and the underlying raw data is both symmetrical and isomorphic. Unfortunately, neither is the case.

Figure 3 illustrates the distortive properties associated with suppression ratio transformations. We will argue that although actual response rate data are based upon ratio scale measurement, the $A /(A+B)$ ratios that are computed from these data do not obey the same logic. In fact, suppression ratio values do not even meet the requirement for interval scale measurement. Ultimately, suppression ratios may lend themselves to rank-order comparisons, but little else in the way of quantitative judgments. Consider the case in which baseline rate $=30$ responses $/ \mathrm{min}$. A CS

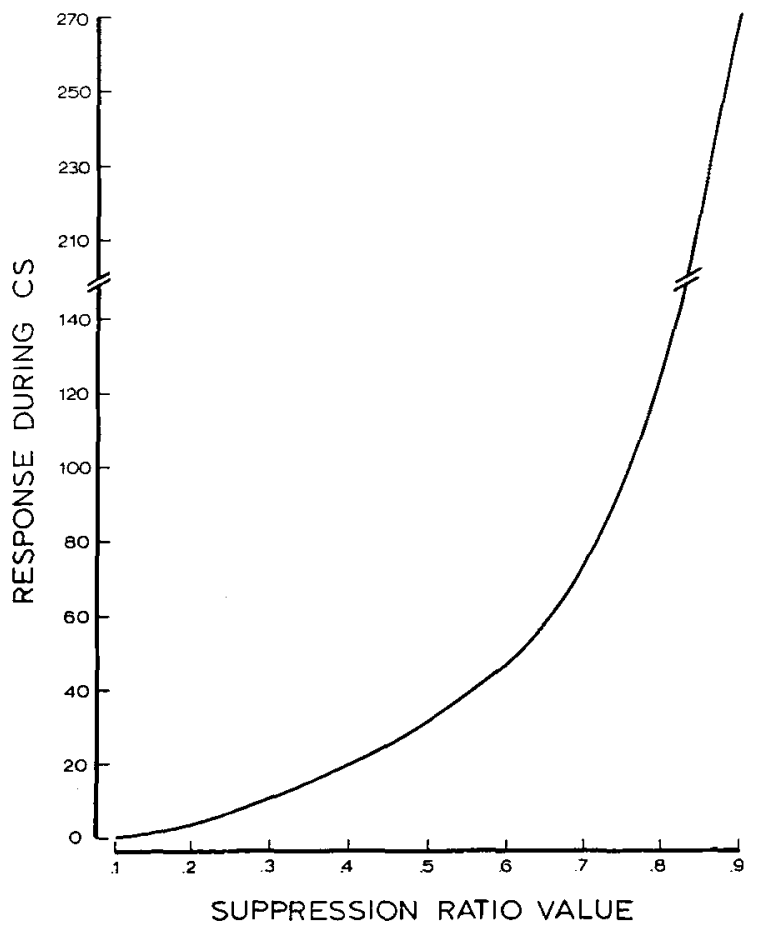

Figure 3. Relationship between the value of $A /(A+B)$ suppression ratio and the rate of responding during CS minutes (A). Calculations are based upon $a$ baseline (B) value $=30$ responses/ min. rate $=30$ yields a ratio of 0.5 . However, consider the increase or decrease in CS responding necessary to move the ratio above or below its resting level of 0.5 . Although it requires only 5 responses in CS to move from a ratio value of .2 to .3 , it requires 24 additional responses in CS to move an equal step from .6 to .7. To move from .8 to .9 requires a further increase of 150 responses.

The asymmetrical and highly skewed nature of the suppression ratio is best demonstrated by considering the change from baseline rate necessary to move in equal steps from ratio values of .1 to .9. These dramatic departures from linearity are illustrated in Figure 3. It should be noted that ratio values above 0.5 , indicating accelerated responding during the CS, are by no means inprobable (Davis et al., 1975, 1976; Davis \& Shattuck, 1980; Hammond, 1966; Hurwitz \& Roberts, 1977).

\section{Inferential Inadequacies of the Suppression Ratio}

It has become customary to evaluate the outcome of conditioned suppression studies by using an analysis of variance (ANOVA) or $t$ test on suppression ratios (Imada, Yamazaki, \& Morishita, 1981; Kamin \& Brimer, 1963; Karpicke, Christoph, Peterson, \& Hearst, 1977; Locurto, Tierney, \& Fitzgerald, 1981; Mackintosh, Bygrave, \& Picton, 1977; Rescorla, 1968; Wagner, Mazur, Donegan, \& Pfantz, 1980). We believe that this kind of analysis is illegitimate.

The ANOVA makes a number of assumptions which are likely to be violated by the use of suppression ratio data. First, it is assumed that the data being tested are normally distributed; secondly, it is assumed that the variances are homogeneous. Ironically, the use of the $A /(A+B)$ transformation loses both of these features, which may have been present in the raw data. There is nothing wrong with ratio transformations per se, nor is their nonlinear relationship to the raw data unique. However, the use of suppression ratios may represent a unique instance in psychology in that normally distributed data such as prime-rate scores or response rates during CS-US have been transformed into nonnormally distributed scores. This is epitomized at the start of Session 1 when normally distributed responses rates are converted into a distribution of ratios with mean $=0.5$ and variance $=0$.

In the likely event that suppressive effects become pronounced on subsequent sessions, the values of $\mathbf{A}$ will be drawn from a highly skewed distribution that is truncated by a limit of zero, a value typically reached by the majority of subjects. Because the distribution of ratio scores will be highly skewed, its variance will be drastically reduced. Of necessity, this results in an inflated likelihood that group differences, however minimal, will be revealed as significant. Thus, in the typical case, the use of ANOVAs on suppression ratio data results in spuriously high significance levels. 
Comparisons involving suppression ratios may also lead to inferential errors in the opposite direction. Specifically, there may be cases in which an ANOVA performed on raw data would have indicated a significant difference, whereas the same analysis performed on suppression ratios would not. By implication, an independent variable may fail to be identified. At first blush, this may seem to be a more conservative strategy and therefore be acceptable. But the situation is more serious, because the failure to detect an independent variable may have considerable theoretical implications.

Consider, for example, a recent experiment by Baker et al. (1981). These investigators concurred in our judgment that baseline rates were affected by conditioned suppression, and thus employed a ratio of the baseline rate of response and the prime rate, that is, $B /(B+P)$. However, their judgment of whether control and experimental conditions differed in baseline rates was founded not upon a comparison of the raw data, but upon an ANOVA of the ratios. The authors failed to detect a difference. Specifically, in their Experiment 2, a "crucial difference" failed to appear between experimental and control groups whose $\mathrm{B} /(\mathrm{B}+\mathrm{P})$ ratios differed by only 0.1 (approximately .5 vs. .6). This small difference, however, masked an approximate $50 \%$ increase in baseline responding, which would likely have yielded a significant difference had raw scores, and not ratios, been analyzed. Not finding this difference, however, prompted Baker et al. to proceed, however wrongly, with the next step in their theoretical progression.

Quite apart from increasing or decreasing the likelihood of significance, there is a far more fundamental reason for not performing ANOVAs on suppression ratio data. As we noted at the outset, the suppression ratio is a relative measure, that is, an index of performance relative to the subject's own baseline. In short, the suppression ratio is an ipsative (as opposed to normative) measure. The case has been repeatedly made that, regardless of their distributional characteristics, ipsative measures should not be tested normatively (Cattell, 1944; Horn, 1963). The logic of this claim may be illustrated by way of analogy. If one wished to compare the weights of men vs. women, it would be appropriate to collect data from a sample of both sexes for direct comparison. It would not be appropriate, however, to form a ratio of each subject's weight relative to his or her ideal weight, and then to compare the sexes on this ratio value. This ipsatized index, while useful for describing individual cases, precludes the direct normative comparison that was originally intended. If one wished to make group comparisons of relative change, there are alternative statistical techniques which focus directly upon difference scores. The advantages of ipsative measurement for describing the behaviors of individual cases are obvious. However, comparisons between cases or between groups of cases should not proceed from this same technique.

\section{CONCLUSION}

Despite extensive research involving the conditioned suppression procedure, we still lack fundmental information. We do not know, for example, which variables are related to the U-shaped function in baseline rate of response. Our own data suggest that CS-US density affects this measure; similarly, there is suggestive evidence that on-line versus offline training, as well as US intensity and CS duration, may affect both the decline and the recovery of baseline responding. It is notable that each of these variables is also presumed to affect conditioned suppression itself, as inferred from the ratio (Blackman, 1977; Davis, 1968; Lyon, 1968; Hurwitz \& Roberts, 1977). In retrospect, it is not possible to establish whether the primary suppressive effect of these variables was on the CS or on the baseline.

To the extent that we continue to employ behavior as an index of a central state, such as "fear," we have demonstrated that the conventional suppression ratio is inappropriate. In fact, we suggest that there are no circumstances in which use of the $A /(A+B)$ index is legitimate. We have identified inadequacies of both a descriptive and an inferential nature which argue in support of our position.

We had hoped to provide a single alternative to the ubiquitous suppression ratio, but this does not seem possible. What, then, do we propose as an alternative strategy for the measurement of conditioned suppression?

Turning first to description, we hold to the view that conditioned suppression is essentially a differentiation phenomenon: the CS exerts Pavlovian control over unknown responses (which have conveniently and intuitively been classified as "fear"). These responses, in turn, modulate the behavior originally under observation. The problem occurs in attempting to express the relative nature of conditioned suppression. We must convey a full complement of information, which includes prime rate data that are necessary to assess session baseline data, which, in turn, are necessary to assess changes during the CS. We do not believe it is possible to combine these measures into a single meaningful index of conditioned suppression. That many investigators are remiss in providing all of these basic data is quite clear. The inevitable consequence is that much, if not all, of the existing literature is uninterpretable.

With regard to statistical inference, we are obliged to offer some substitute for the prevailing method of performing ANOVAs on suppression ratios. As we have noted, it is not the ANOVA, but rather the suppression ratio that is problematic. It is thus reasonable to compare raw data using an ANOVA, pro- 
vided these data do not violate the assumptions of the test. Unfortunately, this may rarely be the case; even the data from our own simple experiment failed to support assumptions of normality or homogeneity of variance. In cases in which response rates are excessively skewed or nonnormal, there is a range of conventional nonparametric tests, such as those employed in the present experiment.

Another approach to analyzing conditioned suppression data involves the use of an analysis of covariance (ANOCOVA) procedure in which raw treatment data are covaried with preexperimental test scores (P). In this manner, it is possible to determine the degree to which later performance reflects the influence of current variables and not preexisting individual differences in responding. Thus, the ANOCOVA satisfies the need for adjustment of the raw scores without compromising the validity of between-subject or between-group comparisons. The ANOCOVA may be a particularly sensitive way to assess both individual and group differences in conditioned suppression because, as Huck (1972) notes, the ANOCOVA "explains away" within-group variability due to pretreatment differences.

Finally, an inferential strategy exists for assessing the degree of conditioned suppression in individual cases. Because successful suppression should be differentially conditioned, it is appropriate to compare the $A$ and $B$ components of the suppression ratio using a $t$ test for related measures. To the extent that these response rates differ, suppression has been differential, as it should be. We have employed this technique on the present data and discovered an unexpected difference. Further precedent for this inferential technique may be found in Davis et al. (1981).

Because conditioned suppression retains relevance to a broad range of issues (Davis \& Hurwitz, 1977), there is no doubt that it is a phenomenon worth further investigation. It would therefore seem that problems associated with its measurement, rather than its "causes," should be given the highest priority by investigators in the field.

\section{REFERENCE NOTES}

1. Fowler, H. Personal communication, November 1981.

2. Ayers, J. J. B. Personal communication, November 1981.

\section{REFERENCES}

AnNaU, Z., \& Kamin, L. The conditioned emotional response as a function of intensity of the US. Journal of Comparative and Physiological Psychology, 1961, 54, 428-432.

Ayres, J. J. B., Berger-Gross, P., Kohler, E. A., Mahoney, W. J., \& STONE, S. Some orderly nonmonotonicities in the trialby-trial acquisition of conditioned suppression. Animal Learning \& Behavior, 1979, 7, 174-180.

Baken, A. G., \& Mackintosh, N. J. Pre-exposure to the CS alone, US alone, or CS and US uncorrelated: Latent inhibition, blocking by context or learned irrelevance? Learning and Motivation, 1979, 10, 278-294.

Baker, A. G., \& Mercier, P. Manipulation of the apparatus and response context may reduce the US pre-exposure interference effect. Quarterly Journal of Experimental Psychology: Comparative and Physiological Psychology, 1982, 34, 221-234.

Baker, A. G., Mercieh, P., Gabel, J., \& Baker, P. A. Contextual conditioning and the US pre-exposure effect in conditioned fear. Journal of Experimental Psychology: Animal Behavior Processes, 1981, 7, 109-128.

Blackman, D. Conditioned suppression and the effects of classical conditioning on operant behavior. In W. K. Honig \& J. E. R. Staddon (Eds.) Handbook of operant behavior. Englewood Cliffs, N.J: Prentice-Hall, 1977.

CatTel., R. B. Psychological measurement: Ipsative, normative and interactive. Psychological Review, 1944, 51, 292-303.

Davis, H. Conditioned suppression: A survey of the literature. Psychonomic Monograph Supplements, 1968, 2(Whole No. 30), 283-291.

DAvis, H. On the importance of operant baselines in studies of operant-Pavlovian interactions. In C. M. Bradshaw, E. Szabadi, \& C. F. Lowe (Eds.), Quantification of steady-state operant behavior. Amsterdam: Elsevier, 1981.

DAvis, H., \& Hurwitz, H. M. B. Operant-Pavlovian interactions. Hillsdale, N.J: Erlbaum, 1977.

Davis, H., \& Levine, S. Predictability, control and the pituitaryadrenal response in rats. Journal of Comparative and Physiological Psychology, 1982, 96, 393-404.

Davis, H., \& McInTine, R. Conditioned suppression under positive, negative, and no contingency between conditioned and unconditioned stimuli. Journal of the Experimental Analysis of Behavior, 1969, 12, 633-640.

Davis, H., MEмmotT, J., \& Hurwitz, H. M. B. Autocontingencies: A model for subtle behavioral control. Journal of Experimental Psychology: General, 1975, 104, 169-188.

Davis, H., Memmott, J., \& HuRwitz, H. M. B. Effects of signals preceding and following shock on baseline responding during a conditioned suppression procedure. Journal of the Experimental A nalysis of Behavior, 1976, 25, 263-277.

Davis, H., \& Sha truck, D. Transfer of conditioned suppression and conditioned acceleration from instrumental to consumatory baselines. Animal Learning \& Behavior, 1980, 8, 253-257.

Davis, H., Shattuck, D., \& Whight, J. Autocontingencies: Factors underlying control of operant baselines by compound tone/shock/no-shock contingencies. Animal Learning \& Behavior, 1981, 9, 322-331.

DAvis, H., \& WRIGHT, J. A note on procedural variability in studies of conditioned suppression. Bulletin of the Psychonomic Society, 1979, 14, 149-150.

Dickinson, A., Colwill, R. M., \& Penace, J. M. Post-trial stimulation and the acquisition of conditioned suppression in the rat. Quarterly Journal of Experimental Psychology, 1980, 32, 149-158.

Dickinson, A., Hall, G., \& Mackintosh, N. J. Surprise and attenuation of blocking. Journal of Experimental Psychology: Animal Behavior Processes, 1976, 2, 313-322.

Estes, W. K., \& Skinnen, B. F. Some quantitive properties of anxiety. Journal of Experimental Psychology, 1941, 29, $390-400$.

Hammond, L. J. Increased responding to CS- in differential CER. Psychonomic Science, 1966, 5, 337-338.

HoRN, J. L. Equations representing combinations of components in scoring psychological variables. Acta Psychologica, 1963, 21, 184-217.

Huck, S. W. The analysis of covariance: Increased power through reduced variability. Journal of Experimental Education, 1972, 41, 42-46.

Hurwitz, H. M. B., \& Roberts, A. E. Aversively controlled behavior and the analysis of conditioned suppression. In $\mathbf{H}$. Davis \& H. M. B. Hurwitz (Eds.), Operant-Pavlovian interactions. Hillsdale, N.J: Erlbaum, 1977. 
Imada, H., Yamazaki, A., \& Morishita, M. The effect of signal intensity upon conditioned suppression: Effects upon responding during signal and intersignal intervals. Animal Learning \& Behavior, 1981, 9, 269-274.

KAmin, L. J., \& Brimer, C. J. The effects of intensity of conditioned and unconditioned stimuli on a conditioned emotional response. Canadian Journal of Psychology, 1963, 17, 194-200.

Karpicke, J., Christoph, G., Peterson, G., \& Hearst, E. Signal location and positive versus negative condition suppression in the rat. Journal of Experimental Psychology: Animal Behavioral Processes, 1977, 3, 105-118.

Locurto, C. M., Tierney, J., \& Fitzgerald, S. Omission training and positive conditioned suppression in the rat. Animal Learning \& Behavior, 1981, 9, 261-268.

LyoN, D. O. Conditioned suppression: Operant variables and aversive control. Psychological Record, 1968, 18, 317-338.

Mackintosh, N. J., Bygrave, D. J., \& Picton, B. M. B. Locus of the effect of a surprising reinforcer in the attenuation of blocking. Quarterly Journal of Experimental Psychology, 1977, 29, 327-336.

Millenson, J., \& Dent, J. G. Habituation of a conditioned emotional response. Quarterly Journal of Experimental Psychology, 1971, 23, 126-134.

Millenson, J., \& DeVilliens, P. A. Motivational properties of conditioned suppression. Learning and Motivation, 1972, 3, 125-137.

Randich, A., \& LoLondo, V. M. Preconditioned exposure to the unconditioned stimulus affects the acquisition of a conditioned emotional response. Learning and Motivation, 1979, 10, 245-277.
Randich, A., \& Rescorla, R. A. The effects of separate presentations of the US on conditioned suppression. Animal Learning and Behavior, 1981, 9, 56-64.

Rescorla, R. A. Probability of shock in the presence and absence of CS in fear conditioning. Journal of Comparative and Physiological Psychology, 1968, 66, 1-5.

Sidman, M. Tactics of scientific research. New York: Basic Books, 1960.

Skinne n, B. F. The behavior of organisms. New York: AppletonCentury-Crofts, 1938.

Srinner, B. F. Science and human behavior. New York: Macmillan, 1953.

Wagner, A. R., Mazur, J. E., Donegan, N. H., \& Pfantz, P. C. Evaluation of blocking and conditioned inhibition to a CS signaling a decrease in US intensity. Journal of Experimental Psychology, 1980, 6, 376-385.

\section{NOTE}

1. There are exceptions, however. There is some suggestion that the degree of baseline suppression may be affected by the nature of the operant reinforcer. Using rats as subjects, both Fowler and Ayers report that no baseline suppression occurred when a licking response was employed (Fowler, Note 1) or when leverpressing was reinforced by sucrose licking (Ayers, Note 2).

(Manuscript received June 16, 1982; revision accepted for publication August 30, 1983.) 